\title{
Pour en lire plus : Le sens écologique
}

\section{Éloïse Simoncelli-Bourque}

\section{(2) OpenEdition}

Journals

Édition électronique

URL : https://journals.openedition.org/ere/8322

ISSN : 2561-2271

\section{Éditeur}

Centr'ERE

\section{Référence électronique}

Éloïse Simoncelli-Bourque, "Pour en lire plus : Le sens écologique », Éducation relative à l'environnement [En ligne], Volume 17-1 | 2022, mis en ligne le 27 janvier 2022, consulté le 02 février 2022. URL : http:// journals.openedition.org/ere/8322

Ce document a été généré automatiquement le 2 février 2022.

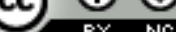

La revue Éducation relative à l'environnement est mise à disposition selon les termes de la Licence Creative Commons Attribution - Pas d'Utilisation Commerciale 4.0 International. 


\title{
Pour en lire plus : Le sens écologique
}

\author{
Éloïse Simoncelli-Bourque
}

\section{RÉFÉRENCE}

Sébastien Bohler (2020). Où est le sens ? Paris : Éditions Robert Laffont, collection "Sciences humaines \& sociales", 384 pages

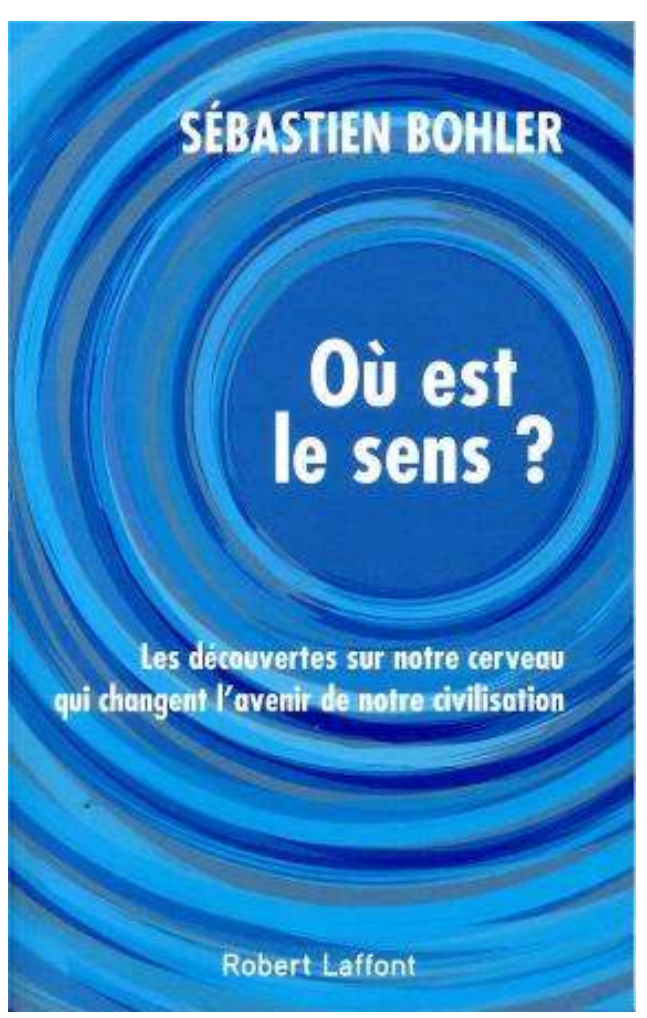


1 Alors que les différents partis politiques provinciaux formulent leur promesse de pourcentage de réduction de gaz à effet de serre en vue des prochaines élections, promesses que certains qualifient d'irréalistes, il m'a semblé important de partager les thèses de Sébastien Bohler sur notre incapacité actuelle à relever le défi climatique. Dans ses deux ouvrages: Le bug humain et où est le sens?, le journaliste et neuroscientifique analyse les rouages de notre cerveau.

2 En s'appuyant sur de nombreuses études, l'auteur explique comment le striatum, partie ancestrale de notre cerveau, fruit de millions d'années d'évolution, siège du circuit de la récompense, répond à cinq mécanismes - manger, avoir des rapports sexuels, être attentif à la moindre information, s'élever dans la hiérarchie sociale et être efficace (agir en dépensant le moins d'énergie possible) - qui ont favorisé la survie de l'homme préhistorique. On comprend aisément que la sélection naturelle ait favorisé les cerveaux qui stimulent le circuit de la récompense afin d'induire ces comportements, essentiels à la reproduction et à la survie de l'espèce en milieu hostile. Cependant, le cerveau de l'homme postmoderne n'a pas évolué au rythme de ses inventions et répond aujourd'hui de la même façon que celui de l'homme préhistorique.

Or la surabondance de nourriture et d'informations, l'accessibilité illimitée à la pornographie (le tiers de l'ensemble de l'activité sur Internet), la valorisation sociale par la consommation et par les réseaux sociaux, ainsi que toutes les inventions qui facilitent le quotidien, suralimentent désormais ces mécanismes. Notre cerveau exigeant toujours plus de dopamine s'enfonce dans ce cercle vicieux de consommationrécompense-consommation. Ainsi, bien que nous soyons de plus en plus conscients de l'ampleur du défi climatique et de l'urgence d'adopter un mode de vie frugal, notre striatum, vieux de plusieurs millions d'années, nous maintient, à coups de dopamine, au mieux dans le statu quo, au pire dans une surconsommation exponentielle et destructrice. En conséquence, le cortex cingulaire, siège de l'intelligence et de la raison, fait face à une dissonance cognitive. Cette incohérence insoutenable entre raison et pulsions et ce sentiment d'impuissance face à notre destin, nous les dissolvons par la fuite en avant, en nous réfugiant dans des mondes virtuels, en débattant de controverses anecdotiques, en faussant nos perceptions et en les transformant en postvérités, en opérant un repli identitaire ainsi qu'en s'abrutissant de divertissement.

4 La postmodernité nous a coupés d'un sens collectif. Les premiers humains avaient développé une compréhension du monde, des origines, des phénomènes naturels, des animaux à travers un sens cosmique. Il y a environ 10000 ans, quand les groupes humains comptèrent un certain nombre d'individus au-delà duquel tous n'étaient connus de tous, on bascula vers un sens social. Des rituels collectifs pratiqués en synchronicité apaisaient l'incertitude face aux inconnus et édictaient les normes morales nécessaires au bon fonctionnement des premières méga sociétés. Depuis, le rationalisme scientifique a réprouvé les visions du monde fondées sur la transcendance, rejetant du même coup toute notion de sacré et tout rituel collectif, pourtant nécessaire au besoin d'appartenance et à la création de sens.

5 Étant donné l'urgence climatique, Bohler espère que ses contemporains du troisième millénaire adopteront le sens écologique. Le sentiment de faire «bien » étant vecteur de sens, une humanité qui considérerait la nature comme sacrée, adopterait des comportements écologiques puisque les comportements destructeurs de l'environnement seraient socialement considérés comme étant « mal ». Il en appelle à "éveiller les consciences à la complexité et à la beauté du monde.» Car 
l'émerveillement agit comme un remède contre l'angoisse existentielle paralysante. Sachant que l'empathie, essentielle à l'édification d'un nouveau sens commun, se développe par la résonnance émotionnelle et la synchronie, Sébastien Bohler soutient qu'il faut recréer des rituels réunissant les gens en vrai, et non en virtuel.

6 Bien que certains critiques aient dénoncé les raccourcis scientifiques qu'aurait empruntés l'auteur, notamment dans Le bug humain, il n'en demeure pas moins qu'ausculter l'immobilisme collectif par la lorgnette des mécanismes du cerveau peut nous amener à esquisser des parallèles avec des perceptions anciennes. Par exemple, le tripartisme de l'âme (désirante, ardente et raisonnante) décrit par Platon dans La République et devenu bipartisme (affects et raison) dans Les lois correspond à bien des égards à ce que décrit Bolher. Platon invite à un équilibre harmonieux entre les différentes âmes et à éduquer les citoyens subordonnés à leur âme désirante (plaisirs corporels et insatiables désirs de posséder) dont les comportements seraient destructeurs pour la justice et la cité. Ainsi, promouvoir une éducation visant non seulement la transmission de savoirs relatifs aux enjeux socio-environnementaux du $\mathrm{XXI}^{\mathrm{e}}$ siècle, mais également l'émerveillement face à l'infinie diversité de la nature constituerait un pas important dans la création commune d'un sens écologique.

Devant l'urgence que nous impose le défi sans précédent auquel est confrontée l'humanité, nous n'en sommes plus à discuter de pourcentages réalistes d'émission de gaz à effet de serre. Il faut impérativement revoir les fondements mêmes de notre civilisation et opérer un changement de paradigme. Pour ce faire, il faudrait incessamment prioriser l'éducation relative à l'environnement non seulement dans nos cursus scolaires, mais également dans la formation des enseignants, de manière à embrasser collectivement un sens écologique. Ceci impliquera d'élever la nature au rang de sacré et de consentir à de substantiels renoncements aux formes abusives de « confort matériel». 